4-1-2021

\title{
Increasing care seeking for children with fever among women in Kebbi State
}

Breakthrough RESEARCH

Follow this and additional works at: https://knowledgecommons.popcouncil.org/departments_sbsr-rh How does access to this work benefit you? Let us know!

\section{Recommended Citation}

Breakthrough RESEARCH. 2021. "Increasing care seeking for children with fever among women in Kebbi State," infographic. Washington, DC: Population Council. 


\section{Increasing Care Seeking for Children with Fever Among Women in Kebbi State}

The Breakthrough RESEARCH project conducted a behavioral surveillance survey for health among women who had a pregnancy or birth in the previous two years.

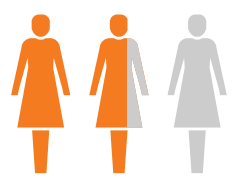

Nearly 2 out of $\mathbf{3}$ (59\%) women in Kebbi State sought care from a formal medical provider for their child under two years of age with fever.

\section{Survey Findings for Kebbi State}

The behavioral surveillance survey identified three important factors for improving care seeking from a formal medical provider for children with fever. The percentages shown below are the averages for all the LGAs sampled. These averages may differ from the median values shown on the next page.

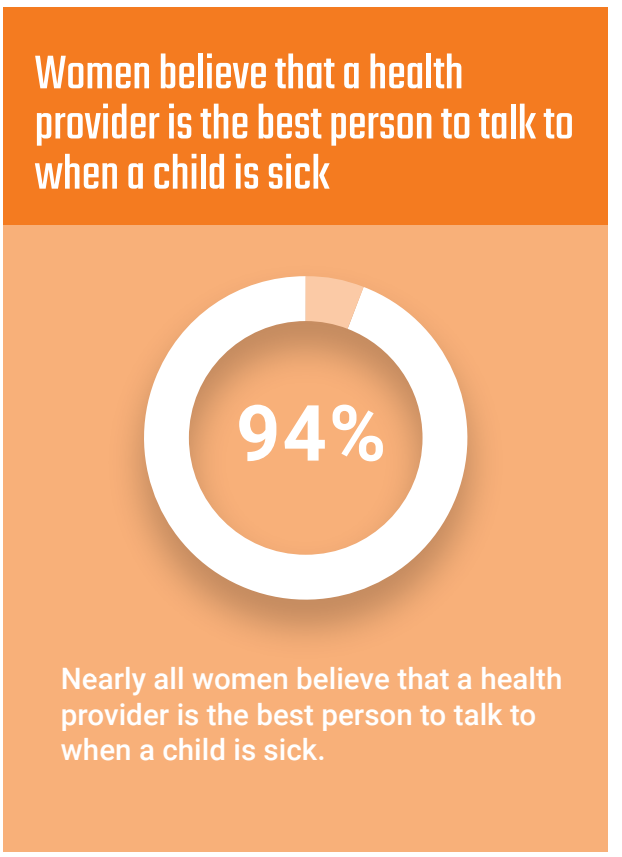

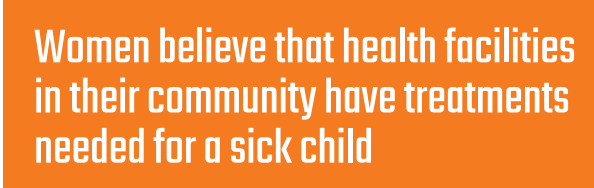

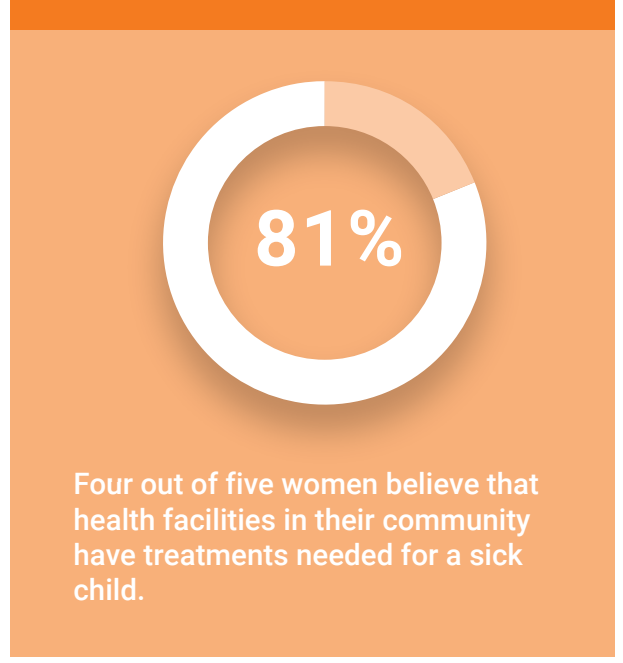

Women are confident that their spouse will allow them to seek advice or treatment for a sick child

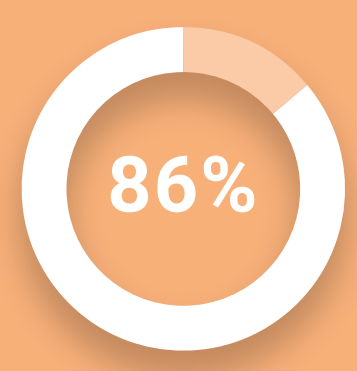

More than four out of five women are confident that their spouse will allow them to seek advice or treatment for a sick child.

\section{If these and other factors are improved further, care-seeking may rise in Kebbi State.}

\section{Survey Results by Local Government Areas in Kebbi State}
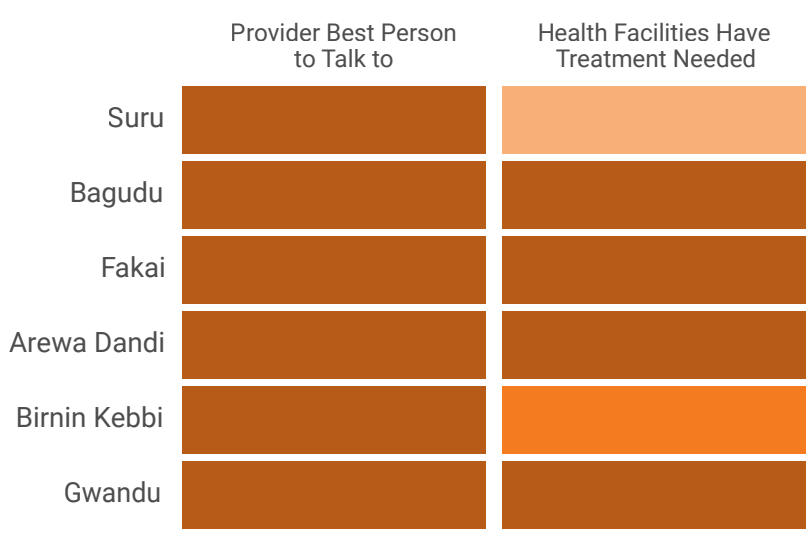

Confident Spouse will Allow Them to Seek Care

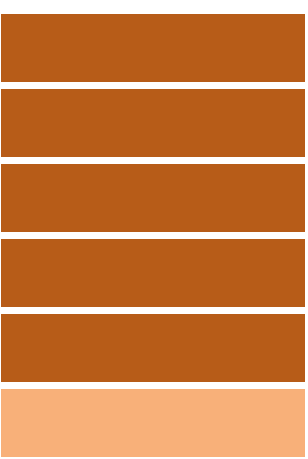

Care Seeking for Child with Fever

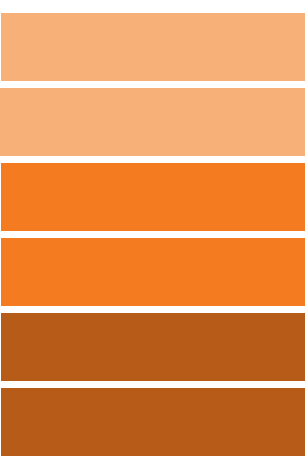

Percentage of women who answered positively, by LGA

$$
\begin{aligned}
& 0 \%-24 \% \\
& \hline 25 \%-49 \% \\
& \hline 50 \%-74 \% \\
& 75-100 \%
\end{aligned}
$$




\section{Increasing Care Seeking for Children with Fever Among Women in Kebbi State}

The Breakthrough RESEARCH project conducted a behavioral surveillance survey for health among women who had a pregnancy or birth in the previous two years.

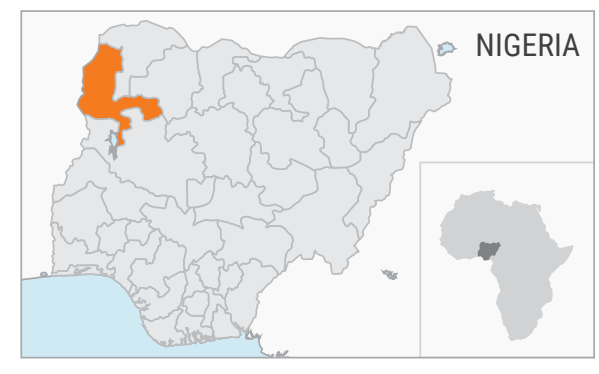

\section{Survey Findings for Kebbi State}

The behavioral surveillance survey identified three important factors for improving care seeking from a formal medical provider for children with fever. The percentage in each box below is the median (50th percentile). Half of local government authorities (LGAs) are below (red) and half above this value (green).

\section{Women believe that a health provider is the best person to talk to when a child is sick}

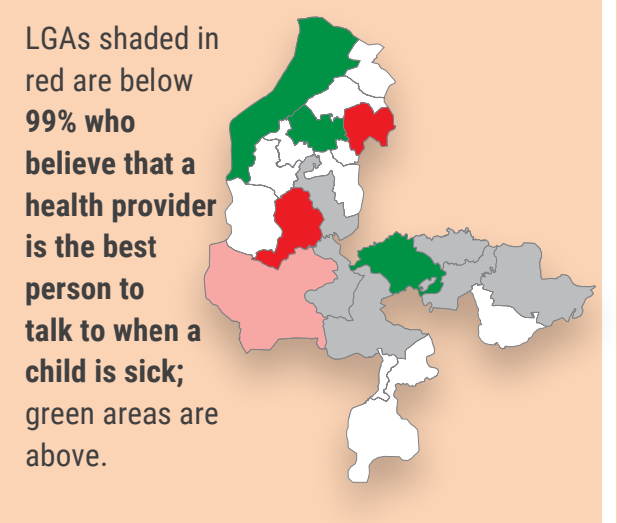

\section{Women believe that health facilities in their community have treatments needed for a sick child}

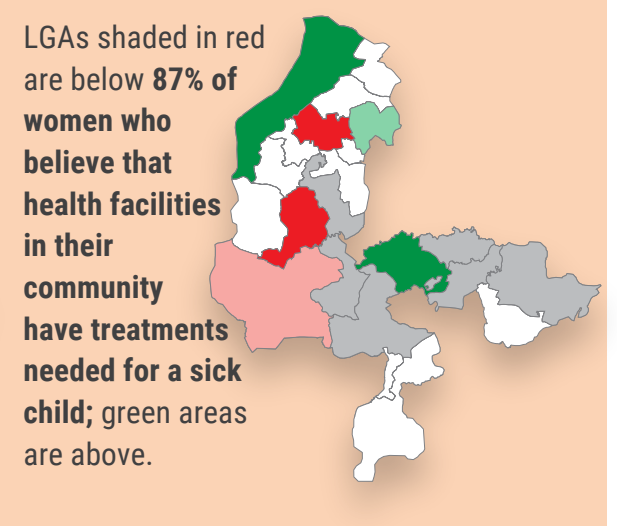

\section{Women are confident that their spouse will allow them to seek advice or treatment for a sick child}

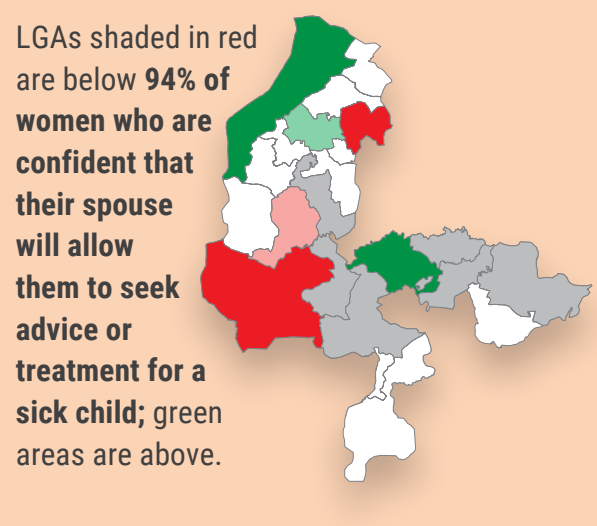

Notes: Lightly shaded areas are immediately below or above the median. Darker shaded areas are further away from the median, below the 25 th or above the 75th percentile. LGAs in white were not sampled for the survey and information is not available for these areas. LGAs in grey have too few women interviewed to reliably estimate the percentage.

\section{How can this information be used to improve care seeking for child fever in Kebbi State?}

Discussion and inquiry into barriers and facilitators of care seeking for child fever may provide insights on how to enhance efforts in these areas by asking questions such as:

- What is different about the wards in LGAs in green that may explain the quality of service delivery in their health facilities? Do LGAs in green receive greater external support for supplies, service quality improvement and social mobilization?

- What is different about the health facilities in the LGAs that may explain how women perceive health services? Are health facilities better resourced and managed? Are providers giving better client services and counseling?

- What is different about the communities that may explain how well an LGA is doing? Do they have stronger leaders, ward development committees, or levels of community engagement in health issues?
- What barriers are present in LGAs in red that inhibit women from seeking formal medical care when their child has a fever? How can these barriers be addressed by community leaders and members of the community?

- Do LGAs in green have facilitators that increase women's ability to seek formal medical care for their child's fever? How can they be adopted in other areas with lower levels of care-seeking?

- How can additional efforts be made or information tailored to reach communities and households in the greatest need of improving these factors related to care-seeking?

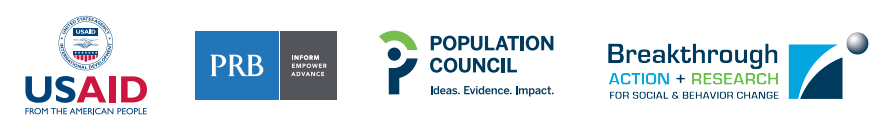

This document is made possible by the generous support of the American people through the United States Agency for International Development (USAID) under the terms of the Breakthrough RESEARCH Project (No. AID-0AA-A-17-00018). The contents are the responsibility of Breakthrough ACTION + RESEARCH and do not necessarily reflect the views of USAID or the United States Government. 